\title{
Alterations in expression levels of genes in p53-related pathways determined using RNA-Seq analysis in patients with breast cancer following CIK therapy
}

\author{
ZUOWEI HU $^{1 *}$, XIAOYE ZHANG ${ }^{2 *}$, HANG YANG $^{1}$, SHUANGLAI QIN $^{1}$, YAQI LIU ${ }^{1}$, \\ WEI XIONG ${ }^{3}$, BING YUAN $^{3}$, LIPING LI $^{4}$, WEIQI YAO ${ }^{3,5,6}$ and DONGCHENG WU ${ }^{2,3}$ \\ ${ }^{1}$ Department of Oncology, Wuhan Integrated TCM and Western Medicine Hospital, Wuhan, Hubei 430022; \\ ${ }^{2}$ Department of Biochemistry and Molecular Biology, Wuhan University, Wuhan, Hubei $430072 ;{ }^{3}$ Department of \\ Research, Wuhan Hamilton Biotechnology Co., Ltd., Wuhan, Hubei 430075; ${ }^{4}$ Department of Oncology, \\ The First People's Hospital of Xiantao, Xiantao, Hubei 433000; ${ }^{5}$ Department of Oncology, Renmin Hospital \\ of Wuhan University; ${ }^{6}$ Wuhan Institute of Biotechnology, Wuhan, Hubei 430060, P.R. China
}

Received May 20,2016; Accepted April 13, 2017

DOI: $10.3892 / \mathrm{ol} .2017 .7205$

\begin{abstract}
The present study aimed at investigating the underlying molecular mechanisms for patients following cytokine-induced killer (CIK) therapy, particularly involving the alterations in $\mathrm{p} 53$-associated signaling pathways, to elucidate whether CIK therapy serves a function in cancer treatment. Samples of blood were collected from patients with breast cancer prior to and following CIK therapy. Two group samples were used for RNA sequencing (RNA-Seq) to determine the alterations in gene expression levels following CIK therapy and one for the quantitative polymerase chain reaction (qPCR), to analyze the reliability of RNA-Seq results. The genes that may encode proteins associated with p53 pathways were selected and analyzed. The expression levels of 8 genes were analyzed, including tumor suppressor protein 53 (TP53), murine double minute homolog 2 (MDM2), ribosomal protein L11 (RPL11), ribosomal protein S23 (RPS23), sirtuin 1 , histone deacetylase 1 , tuberous sclerosis complex 1 (TSC1) and mechanistic target of rapamycin (mTOR), and alterations in expression levels following CIK therapy were determined. However, only RPL11 and RPS23 were identified to exhibit marked alterations in expression levels (FDR $<0.05$ ), which was considered to be due to individual distinctions. $\mathrm{qPCR}$
\end{abstract}

Correspondence to: Dr Weiqi Yao or Dr Dongcheng Wu, Department of Research, Wuhan Hamilton Biotechnology Co., Ltd., B6-423, 666 High-Tech Avenue, Donghu New Technology Development Zone, Wuhan, Hubei 430075, P.R. China

E-mail: weiqiyaodoc@163.com

E-mail: jiazhanghuang@yeah.net

\section{${ }^{*}$ Contributed equally}

Key words: breast cancer, cytokine-induced killer therapy, p53, murine double minute 2 homolog, RNA sequencing analysis revealed that the expression levels of the RPL11, TP53 and TSC1 genes were downregulated, and those of the RPS23 and MDM2 genes were upregulated following CIK therapy. Only MDM2 exhibited a marked alteration in the gene expression level following CIK therapy. Alterations in the expression levels of TP53, RPL11 and TSC1 were associated with those of MDM2, RPS23 and mTOR, respectively.

\section{Introduction}

Breast cancer is the most frequently diagnosed cancer in females and is currently the second most common cause of cancer-associated mortality behind lung cancer $(1,2)$. The American Cancer Society estimated that, in the USA, 40,290 of 231,840 patients with newly diagnosed invasive breast cancer would succumb to breast cancer in 2015 (3). It was also predicted that, in $2015,<12 \%$ of females in the USA would develop invasive breast cancer over their lifetime (4). One type of breast cancer, triple-negative breast cancer (TNBC), is characterized by the absence of the following genes: Estrogen receptor $\alpha(E R \alpha)$, progesterone receptor and human epidermal growth factor receptor 2 (HER2) (5). These genes are common targets for therapy, which makes treatment of TNBC a challenge, due to the lack of expression of these target genes in TNBC patients (5). Patients with TNBC are considered to possess poorer clinical outcomes and exhibit an increased rate of distant recurrence, compared with other types of breast cancer.

Understanding types of cancer has enabled novel and systemic treatments to be offered patients with breast cancer in order to improve their prognosis. Cytokine-induced killer (CIK) therapy is a new and widely used tumor-adoptive immunotherapeutic method which has been combined with surgery, chemotherapy and radiation therapy to enhance the antitumor effects (6-8). Autologous CIK cells have been widely accepted as an effective therapeutic method for solid and hematological malignances. The advantages of this therapy are numerous: CIK therapy exhibits antitumor effects, is easily produced in vitro, 
possesses greater efficacy with fewer side effects compared with radiotherapy or chemotherapy, and major histocompatibility complex-unrestricted cytotoxicity (9). However, further studies are required to investigate the signaling pathway and underlying molecular mechanisms of CIK therapy.

The tumor suppressor and transcription factor p53 is encoded by the tumor protein p53 (TP53) gene, and triggers cell cycle arrest, cell proliferation, apoptosis and senescence in response to a variety of cellular stress signals (10-12). Stress signals activate the transcriptional activity of p53 and may also induce stabilization of the protein through post-translational modifications including phosphorylation (11-13). Degradation of p53 by proteasomes is mediated by the ubiquitination of murine double minute 2 (MDM2) and this process may be enhanced by the interaction of MDM2 and phosphatase and tensin homolog deleted on chromosome 10 (PTEN) (14-16). TP53 gene mutations always result in a loss of wild-type p53 oncosuppressive properties, therefore numerous mechanisms have been studied, including p53 mutations and deletions, and MDM2 amplification. p53 forms a homotetramer prior to serving as a transcription factor and so expression of TP53 mutant variants may lead to novel oncogenic gain-of-function (GOF) activities in cancer cells (17). Previous studies have demonstrated that the incidence of p53 mutations differs between various types of cancer, ranging from near universal ( $\sim 96 \%$ for serous ovarian cancer) to rare occurrence $(<10 \%$ for thyroid cancer) (18). The incidence of p53 mutations is between 18 and $25 \%$ for primary breast carcinomas (19). It has also been demonstrated that GOF mutant p53 may be able to upregulate CXC chemokines and enhance cell migration (20-23).

MDM2, also known as human double minute (HDM) 2 in humans, is a negative regulator of p53 and may promote p53 degradation by binding to the protein using its N-terminal p53-binding domain (24). At the C-terminus of MDM2, the really interesting new gene (RING) domain is responsible for p53 degradation, serving as an E3 ligase and aiding the formation of polyubiquitin chains (23). MDM2 is also able to inhibit the transcriptional activation of p53 by binding to the protein at the $\mathrm{N}$-terminus and promoting p53 degradation (25). In a number of human cancers, MDM2 is overexpressed, and is associated with poor prognosis in cancers including sarcoma, acute lymphocytic leukemia and glioma (26). MDM2 may interfere with p53-mediated apoptosis in tumors (27) and cause carcinogenesis independently of the p53 signaling pathway (25). Previous studies have identified that loss of MDM2 mimics the inhibition of the MDM2-p53 interaction and increases the stabilization of mutant p53 and the incidence of metastasis $(28,29)$.

Although CIK therapy is administered to patients with breast cancer, and accepted as a form of therapy, the molecular mechanisms and pathways of CIK therapy remain unclear. Previously, it was proposed to use RNA sequencing (RNA-Seq) to detect the gene expression changes in 2 patients with breast cancer (Zhou et al, unpublished data). Samples from a third patient with breast cancer were analyzed using the quantitative polymerase chain reaction (qPCR) to assess the reliability of RNA-Seq results. Samples of blood were collected prior to and following CIK therapy from the patients with breast cancer. In the present study, the expression levels of 9 genes were determined [TP53, p85, MDM2, MDM4, ribosomal protein L11
(RPL11), ribosomal protein S23 (RPS23), sirtuin 1 (SIRT1), histone deacetylase 1 (HDAC1), tuberous sclerosis complex 1 (TSC1) and mechanistic target of rapamycin (mTOR)]. The expression levels of genes which may regulate the expression or activity of p53 (8/9 genes aforementioned) were determined using RNA-Seq. MDM2 only demonstrated a marked alteration in expression levels subsequent to CIK therapy For the genes that were able to regulate the expression or activities of p53, the expression levels of 8 genes out of 9 (except p85) were determined using RNA-Seq, with only MDM2 identified to be significantly altered in expression levels following CIK therapy. The present study demonstrated that in a number of breast cancers, alterations in the expression of TP53 were associated with alterations in the expression of MDM4. This pattern of association was also observed between the RPL11 and RPS23 genes, and the TSC1 and mTOR genes.

\section{Materials and methods}

Patient samples. In the present study, mononuclear cells were collected from three female patients with breast cancer (age range, 37-55 years) in March 2016 in Wuhan Integrated TCM and Western Medicine Hospital (Wuhan, China), 2 weeks after the patients had completed chemotherapy or radiation. The cells were separated from the patient's peripheral blood and cultured for 14 days, following which the CIK cell number, viability and phenotype were determined to confirm safety for infusion. Patients were administered three intravenous infusions of $>5 \times 10^{9} \mathrm{CIK}$ cells at 1 day intervals. The peripheral blood from 3 patients with breast cancer was collected prior to and following CIK therapy. The present study was approved by the ethical review board of the Wuhan Integrated TCM and Western Medicine Hospital (Wuhan, China), and written informed consent was obtained from all participants.

cDNA preparation. Peripheral blood, collected from the patients, was first separated using lymphocyte separation medium (GE Healthcare Bio-Sciences, Pittsburgh, PA, USA) and lymphocytes were collected for cDNA preparation. TRIzol (Invitrogen; Thermo Fisher Scientific, Inc., Waltham, MA, USA), trichloromethane, isopropanol and ethyl alcohol, were used to extract RNA, according to the manufacturer's protocol (Invitrogen; Thermo Fisher Scientific, Inc.). A total of $40 \mu \mathrm{l}$ nuclease-free water was used to resuspend RNA and a NanoDrop 2000 instrument (Thermo Fisher Scientific, Inc.) was used to determine the quantity and purity of RNA. The absorbance at $260 \mathrm{~nm}$ was determined and the absorbance ratio at 260/280 was calculated. Finally, a Moloney murine leukemia virus Reverse Transcriptase kit (Promega Corporation, Madison, WI, USA) was used to generate the final cDNA library from the total extracted RNA.

Two paired samples were selected for RNA-Seq, termed L_1 (prior to CIK therapy) and L_2 (following CIK therapy) for one patient and $Z_{-} 1$ (prior to CIK therapy) and $Z_{-} 2$ (following CIK therapy) for the other patient. Samples from another patient were used for qPCR analysis.

qPCR validation. qPCR was conducted to re-analyze selected genes listed in Table I and to determine the validity of sequencing data. PCR amplifications were performed using 
Table I. Primers used in the quantitative polymerase chain reaction.

\begin{tabular}{lll}
\hline Gene & \multicolumn{1}{c}{ Forward $\left(5^{\prime}-3^{\prime}\right)$} & \multicolumn{1}{c}{ Reverse $\left(5^{\prime}-3^{\prime}\right)$} \\
\hline TP53 & TCAGCATCTTATCCGAGTGGAA & AGGGCACCACCACACTATGTC \\
TSC1 & GAAAGCCGCCTATCGGAAA & TCCGTTTTGGGAGGTATCAAG \\
RPL11 & CGCATCCGCAAACTCTGTCT & TCCGGATGCCAAAGGATCT \\
RPS23 & CGAGACCAGAAGTGGCATGA 2 & GCATGAGAAGCACCTCCAAAAG \\
GAPDH & CAGGCAGGGGAGAGTGATA & GTGATGGAAGGGGGGGATT \\
& CATGAGAAGTATGACAACAGCCT & AGTCCTTCCACGATACCAAAGT
\end{tabular}

GADPH was used as an internal control. TP53, tumor protein 53; TSC1, tuberous sclerosis complex 1; RPL11, ribosomal protein L11; RPS23, ribosomal protein S23; MDM2, murine double minute 2 homolog.

Table II. Detailed information of the MDM2 gene for two patients using RNA sequencing.

\begin{tabular}{lcc}
\hline MDM2 gene & Patient 1 & Patient 2 \\
\hline Fold change & 2.3 & 3.0 \\
$\log _{2}$ (fold-change) & 1.2 & 1.6 \\
P-value & $2.3 \times 10^{-11}$ & $2.3 \times 10^{-3}$ \\
FDR & $5.9 \times 10^{-11}$ & $4.6 \times 10^{-3}$ \\
Expression change & $\mathrm{Up}$ & $\mathrm{Up}$ \\
Expression following therapy & 40.9 & 44.0 \\
Expression prior to therapy & 17.8 & 14.5 \\
Description & E3 ubiquitin-protein ligase MDM2 & E3 ubiquitin-protein ligase MDM2 \\
\hline
\end{tabular}

FDR, false discovery rate; hsa, human; MDM2, murine double minute 2 homolog.

200 ng cDNA, $10 \mu 1 \mathrm{SYBR}^{\circledR}$ qPCR Mix (CWBIO, Beijing, China) and $0.4 \mu \mathrm{l}(10 \mu \mathrm{M})$ primers (Table I). For each gene tested, 3 replicates were performed and the GAPDH gene was used as the internal reference gene. RNase-free $\mathrm{H}_{2} \mathrm{O}$ (CWBIO) was added to the mixture to a final volume of $20 \mu \mathrm{l}$. Following heating to $95^{\circ} \mathrm{C}$ for $10 \mathrm{~min}, 40$ cycles of $5^{\circ} \mathrm{C}$ for $15 \mathrm{sec}, 55^{\circ} \mathrm{C}$ for $30 \mathrm{sec}$ and $72^{\circ} \mathrm{C}$ for $30 \mathrm{sec}$ were applied. The $2^{-\Delta \Delta \mathrm{Cq}}$ method (30) was used for relative gene expression level analysis.

\section{Results}

Data from RNA-Seq. From a previous study (Zhou et al, unpublished data), the MDM2 gene was detected in the 2 paired samples using RNA-Seq. Expression changes were demonstrated in the 2 patients prior to and following CIK therapy, with an increase of 2.1-fold for 1 patient and 3.05-fold for the other patient (Table II).

Vijayakumaran et al (31) demonstrated that p53 expression, particularly mutant p53 in cancer cells, may be regulated by several proteins and microRNAs (miRs). The proteins that may control p53 proteasomal degradation include MDM2, MDM4, RPL11, RPS23, p85, cell division control protein 42 (CDC42), SIRT1, HDAC1, TSC1 and the mTOR signaling pathway. In the present study, attempts to identify additional proteins associated with p53, other than MDM2, in CIK therapy were carried out. The data collected using RNA-Seq (Table III) demonstrated alterations in the expression levels of the TP53, MDM4, RPL11, RPS23, SIRT1, HDAC1, TSC1 and mTOR genes identified in the two paired samples. Analysis of results revealed that only the RPL11 and RPS23 pseudogenes were detected with significant alterations in expression [false discovery rate $(\mathrm{FDR})<0.05]$ in the two paired samples. Additionally, marked changes in the expression level of the mTOR gene was determined in one of the paired samples which was possibly due to individual differences.

$q P C R$ validation of gene transcription. To confirm the validity of RNA-Seq data and previous studies (Zhou et al, unpublished data) on p53 regulation, the expression levels of genes, including TP53, MDM2, RPL11, RPS23 and TSC1, were all analyzed by qPCR. The results identified that the RPL11, TP53 and TSC1 genes were all downregulated following therapy for one patient, and the RPS23 and MDM2 genes were upregulated following CIK therapy (Fig. 1).

\section{Discussion}

RNA-Seq and qPCR identified 3 genes, MDM2, TP53 and $\mathrm{TSC} 1$, of the third patient with similar alterations to those of one of the patients with samples termed L_2 and $Z_{-} 2$. The distinctions were possibly due to individual variations. 
Table III. Detailed information of gene expression levels for two patients using RNA sequencing.

\begin{tabular}{lrrrr}
\hline mRNA & L_1 & L_2 & Z_1 & Z_2 \\
\hline CDC42 & 165 & 252 & 389 & 248 \\
HDAC1 & 134 & 294 & 344 & 288 \\
MDM4 & 51 & 120 & 141 & 81 \\
mTOR & 75 & 128 & 147 & 53 \\
RPL11 & 2,045 & 3,260 & 4,589 & 3,790 \\
RPS23 & 490 & 722 & 1,008 & 695 \\
SIRT1 & 8 & 17 & 29 & 18 \\
TP53 & 26 & 119 & 96 & 57 \\
TSC1 & 72 & 105 & 122 & 79 \\
\hline
\end{tabular}

CDC42, cell division control protein 42 homolog; HDAC1, histone deacetylase 1; MDM2, murine double minute 2 homolog; MDM4, mouse double minute 4 homolog; mTOR, mechanistic target of rapamycin; RPL11, ribosomal protein L11; RPS23, ribosomal protein S23; SIRT1, sirtuin (silent mating type information regulation 2 homolog) 1; TP53, tumor suppressor protein 53; TSC1, tuberous sclerosis complex 1 .

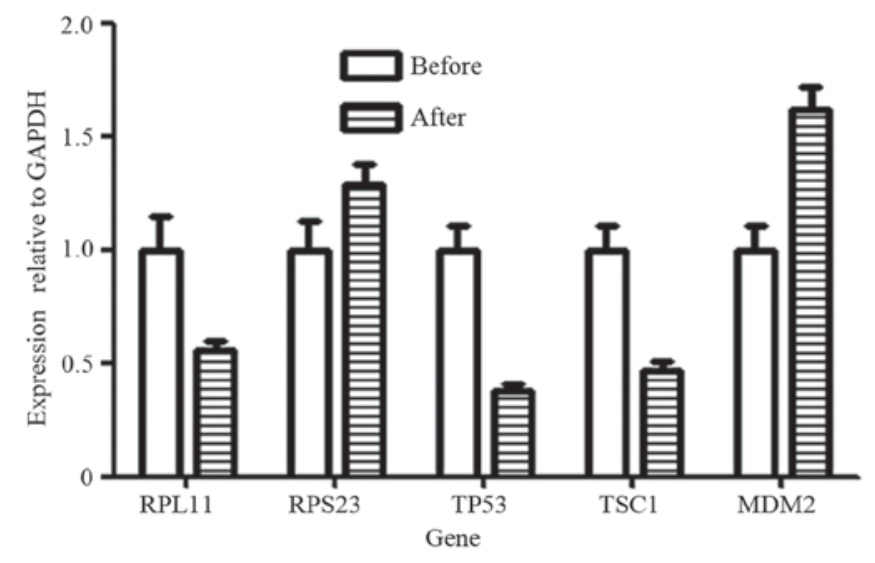

Figure 1. Results of the quantitative polymerase chain reaction. RPL11, ribosomal protein L11; RPS23, ribosomal protein S23; TP53, tumor protein 53; TSC1, tuberous sclerosis complex 1; MDM2, murine double minute 2 homolog.

In normal cells, transcription factor p53 is regulated and maintained at low expression levels by MDM2. As an E3 ubiquitin ligase, MDM2 is able to bind to p53 specifically by its $\mathrm{N}$-terminus, form ubiquitin chains on $\mathrm{p} 53$ by the catalytic RING domain at the MDM2 C-terminus and result in proteasomal degradation of p53 (24). The MDM2-p53 interaction is complex, and is regulated at a number of levels by numerous cellular proteins and epigenetic mechanisms. In $\sim 50 \%$ of human cancers, the gene TP53 is mutated, including deletion, loss-of-function and gain-of function mutations; however, $17 \%$ of tumors exhibit MDM2 gene amplification. Although p53 induces the expression of MDM2, overexpression of MDM2 may promote the degradation and inhibit the cellular activity of p53. Therefore, increased expression levels of MDM2 may lead to the inhibition of MDM2-p53 interactions, which will lead to downregulation of p53 pathways by promoting the degradation of wild-type and mutated p53 proteins.

In the present study, the expression levels of MDM2 were determined by RNA-Seq in the two samples with markedly upregulated alterations following CIK therapy. Similar alterations in the third patient were confirmed by qPCR. The primary function of MDM2 is to promote degradation of $\mathrm{p} 53$, and, therefore, the results of the present study indicated that the signaling pathways and underlying molecular mechanisms of CIK therapy for breast cancer enhanced apoptosis of cells, particularly in cancer cells with p53 mutations. Subsequently, the expression levels of the TP53 gene were analyzed and it was revealed that TP53 was expressed in the two paired samples, with no marked alterations following CIK therapy. However, results from one paired sample demonstrated that the expression of TP53 was upregulated following therapy, whereas the other sample revealed downregulation following treatment. As in $\sim 50 \%$ of human cancers, the TP53 gene is mutated in a number of ways, including simple mutation, deletion, loss-of-function and gain-of function. It was hypothesized that if the patients developed breast cancer due to p53 mutations, the pathways and underlying molecular mechanisms of CIK therapy may be associated to degrade mutant $\mathrm{p} 53$ proteins and to promote apoptosis of cells with the TP53 mutants. This hypothesis, that downregulated expression of p53 was caused by elimination of non-recoverable p53 mutants, requires additional study.

The expression of wild-type p53 may lead to apoptosis of cells by upregulating target genes including p21, B-cell lymphoma 2 (Bcl-2)-like protein, p53 upregulated modulator of apoptosis and phorbol 12-myristate 13-acetate-induced protein 1 (32). However, the pathways and underlying molecular mechanisms of p53-mediated tumor suppression remain unclear. In breast cancers with mutant p53, the signaling pathways associated with $\mathrm{p} 53$ for apoptosis differ from those in normal cells. In a previous study of acute myeloid leukemia, Bcl-2, inhibited by $\mathrm{p} 53$-mediated signaling pathways, may induce apoptosis in mitochondria (33). In addition, the upregulated expression of MDM2 may induce apoptosis through this aforementioned signaling pathway by enhancing the degradation of $\mathrm{p} 53$. These hypotheses suggest additional signaling pathways for CIK therapy.

MDM2 may negatively regulate the transcriptional activity of $\mathrm{p} 53$ by monoubiquitin to trigger $\mathrm{p} 53$ from nuclear exportation (34). It may be an additional signaling pathway mediated by MDM2 and p53 for CIK therapy with non-recoverable p53 mutations.

MDM4 is a primary negative regulator of $\mathrm{p} 53$, similar to MDM2, and is known as MDMX and HDMX. MDM4 is a homologous MDM2 protein that may be induced by $\mathrm{p} 53$ and assist in the maintenance of low $\mathrm{p} 53$ expression levels in normal cells (35). MDM4, similarly to MDM2, is overexpressed in human cancers including sarcoma and breast cancer with incidences of $\sim 20$ and $15 \%$, respectively (17). The MDM2-MDM4 heterodimer is formed via the binding of the C-terminus of the two proteins, although MDM4 does not possess E3 ligase activity at C-terminus, unlike MDM2. MDM4 may enhance ubiquitination on 53 by forming MDM2-MDM4 heterodimers (36) and inhibit the transcriptional activity of p53 by binding to it via its $\mathrm{N}$-terminus (37). In the present study, the 
expression levels of MDM4 were altered in the two paired samples with FDR values $>0.05$, which means that these alterations were not marked. The alterations in MDM4 expression levels were distinct between patients, similar to that observed in TP53, with expression upregulated for one patient and downregulated for another. It was identified that the patient with upregulated expression levels of TP53 additionally exhibited upregulated expression of MDM4 and the other patient exhibited downregulated expression of these two genes. The results demonstrate that, for a number of patients with breast cancer, alterations in the expression levels of TP53 were associated with that of MDM4. Therefore, it was hypothesized that the trend of expression levels observed in patients with cancer following CIK therapy were due to the distinct expression patterns in the MDM4 gene, as MDM4 may enhance p53 degradation by forming MDM2-MDM4 heterodimers (35). The results may validate the previous hypothesis that MDM2 serves a function in CIK therapy, through p53-mediated signaling pathways including leading to apoptosis.

The expression levels of p53, MDM2 and MDM4 may all be regulated by distinct miRs. For example, miR-192, -194, $-215,-143,-145$ and -605 may enhance the expression levels of p53 through the suppression of MDM2 expression, and subsequently the expression levels of these miRs may be upregulated by p53 (38). Additional studies are required to investigate the regulation mechanisms of p53, MDM2 and MDM4 following CIK therapy for patients with cancer.

Expression levels of p53 may be additionally regulated by other proteins, including RPL11, RPS23, p85, CDC42, SIRT1, HDAC1, TSC1 and the mTOR signaling pathway (31). According to a previous study, RPL11 may enhance the expression levels of p53 through inhibiting MDM2, whereas RPS23 may enhance the activity of RPL11 by inducing ribosomal stress (31). In the present study, the expression of the RPL11 and RPS23 genes were determined using RNA-Seq in two paired samples and identified increased expression levels, particularly for the RPL11 gene (Table III). The expression levels of these two aforementioned genes did not markedly alter, with an FDR value $>0.05$. The expression level of RPL11 was upregulated in the two paired samples, whereas the expression level of RPS23 was upregulated for one patient and downregulated for another. The results of the present study revealed that the expression level of RPL11 was markedly altered for one patient when the expression level of RPS23 was upregulated. However, when the expression level of RPS23 was downregulated, the expression levels of RPL11 did not alter as much following therapy. As a ribosomal protein, RPL11 may bind to MDM2 and block its inhibition of p53 (39), which may lead to a novel hypothesis that the expression levels of RPL11 were associated with those of RPS23. Additional studies are required to determine the functions of these aforementioned proteins in p53- and MDM2-mediated pathways for patients with cancer following CIK therapy.

TSC1 may enhance the activities of p53 through inhibition of the mTOR signaling pathway (31). In the present study, the expression levels of two genes, TSC1 and mTOR, were upregulated in one paired sample and downregulated in another patient following CIK therapy. These results suggest that the expression levels of mTOR may be associated with those of TSC1. Elucidation of the function and underlying molecular mechanisms of TSC1 and mTOR in p53-mediated pathways in patients with cancer following CIK therapy requires additional study.

p85, CDC42, SIRT1 and HDAC1 may inhibit the activity of p53 directly. According to the RNA-Seq data, the trends in expression levels of CDC42 and HDAC1 were similar to those of TSC1 and mTOR. The expression levels of SIRT1 were decreased, with upregulation in the two patients. However, no signals were determined with respect to the expression of $\mathrm{p} 85$, suggesting that $\mathrm{p} 85$ may not be expressed in these two patients.

In the present study, expression levels of 9 genes were analyzed, including TP53, MDM2, MDM4, RPL11, RPS23, SIRT1, HDAC1, TSC1 and mTOR. For the genes that may regulate the expression or activity of p53, the expression levels of 8/9 genes were determined by RNA-Seq, with only MDM2 identified as markedly altered following CIK therapy. In addition, the alterations in the expression levels of TP53, RPLI1 and TSC1 were associated with that of MDM4, RPS23 and mTOR, respectively. The aforementioned proteins may regulate the expression and activity of p53 at different levels in signaling pathways. Elucidation of the function and underlying molecular mechanisms of these proteins in p53-mediated signaling pathways in patients with cancer following CIK therapy requires additional study. Furthermore, the expression levels and activity of these proteins are regulated by additional proteins and miRNAs. Additional studies are required to investigate the upstream signaling pathways mediated by the aforementioned 8 genes through the expression changes of target genes for patients with cancer following CIK therapy.

\section{Acknowledgements}

The authors thank the Department of Research, Wuhan Hamilton Biotechnology Co., Ltd. (Wuhan, China), for their assistance, including the provision of laboratory apparatus and assistance in data analyses.

\section{References}

1. American Cancer Society: Cancer facts \& figures 2015. https://www.cancer.org/content/dam/cancerorg/research/cancer-facts-and-statistics/annual-cancer-facts-andfigures/2015/cancer-facts-and-figures-2015.pdf.

2. Edwards BK, Noone AM, Mariotto AB, Simard EP, Boscoe FP, Henley SJ, Jemal A, Cho H, Anderson RN, Kohler BA, et al: Annual Report to the Nation on the status of cancer, 1975-2010, featuring prevalence of comorbidity and impact on survival among persons with lung, colorectal, breast, or prostate cancer. Cancer 120: 1290-1314, 2014.

3. Siegel RL, Miller KD and Jemal A: Cancer statistics, 2015: CA Cancer J Clin 65: 5-29, 2015.

4. Lo-Fo-Wong DN, Sitnikova K, Sprangers MA and de Haes HC: Predictors of health care use of women with breast cancer: A systematic review. Breast J 21: 508-513, 2015.

5. Rakha EA, El-Sayed ME, Green AR, Lee AH, Robertson JF and Ellis IO: Prognostic markers in triple-negative breast cancer. Cancer 109: 25-32, 2007.

6. Li H, Wang C, Yu J, Cao S, Wei F, Zhang W, Han Y and Ren XB: Dendritic cell-activated cytokine-induced killer cells enhance the anti-tumor effect of chemotherapy on non-small cell lung cancer in patients after surgery. Cytotherapy 11: 1076-1083, 2009.

7. Hui KM: CIK cells-current status, clinical perspectives and future prospects-the good news. Expert Opin Biol Ther 12: 659-661, 2012

8. Ma Y, Zhang Z, Tang L, Xu YC, Xie ZM, Gu XF and Wang HX: Cytokine-induced killer cells in the treatment of patients with solid carcinomas: A systematic review and pooled analysis. Cytotherapy 14: 483-493, 2012 
9. Wang X, Yu W, Li H, Yu J, Zhang X, Ren X and Cao S: Can the dual-functional capability of CIK cells be used to improve antitumor effects? Cell Immunol 287: 18-22, 2014.

10. Bartkova J, Horejsí Z, Koed K, Krämer A, Tort F, Zieger K, Guldberg P, Sehested M, Nesland JM, Lukas C, et al: DNA damage response as a candidate anti-cancer barrier in early human tumorigenesis. Nature 434: 864-870, 2005.

11. Harms K, Nozell S and Chen X: The common and distinct target genes of the p53 family transcription factors. Cell Mol Life Sci 61: 822-842, 2004.

12. Zhao Y, Chaiswing L, Velez JM, Batinic-Haberle I, Colburn NH, Oberley TD and St Clair DK: p53 translocation to mitochondria precedes its nuclear translocation and targets mitochondrial oxidative defense protein-manganese superoxide dismutase. Cancer Res 65: 3745-3750, 2005.

13. Chipuk JE, Kuwana T, Bouchier-Hayes L, Droin NM, Newmeyer DD, Schuler M and Green DR: Direct activation of Bax by p53 mediates mitochondrial membrane permeabilization and apoptosis. Science 303: 1010-1014, 2004.

14. Mayo LD and Donner DB: A phosphatidylinositol 3-kinase/Akt pathway promotes translocation of Mdm2 from the cytoplasm to the nucleus. Proc Natl Acad Sci USA 98: 11598-11603, 2001.

15. Gottlieb TM, Leal JF, Seger R, Taya Y and Oren M: Cross-talk between Akt, p53 and Mdm2: Possible implications for the regulation of apoptosis. Oncogene 21: 1299-1303, 2002.

16. Oren M, Damalas A, Gottlieb T, Michael D, Taplick J, Leal JF, Maya R, Moas M, Seger R, Taya Y and Ben-Ze'ev A: Regulation of p53: Intricate loops and delicate balances. Biochem Pharmacol 64: 865-871, 2002.

17. Shtraizent N, Matsui H, Polotskaia A and Bargonetti J: Hot spot mutation in TP53 (R248Q) causes oncogenic gain-of-function phenotypes in a breast cancer cell line derived from an African American patient. Int J Environ Res Public Health 13: ijerph13010022, 2015

18. Burgess A, Chia KM, Haupt S, Thomas D, Haupt Y and Lim E: Clinical Overview of MDM2/X-Targeted Therapies. Front Oncol 6: 7, 2016.

19. Alsner J, Yilmaz M, Guldberg P, Hansen LL and Overgaard J: Heterogeneity in the clinical phenotype of TP53 mutations in breast cancer patients. Clin Cancer Res 6: 3923-3931, 2000

20. Freed-Pastor WA and Prives C: Mutant p53: One name, many proteins. Genes Dev 26: 1268-1286, 2012.

21. Walerych D, Napoli M, Collavin L and Del Sal G: The rebel angel: Mutant p53 as the driving oncogene in breast cancer. Carcinogenesis 33: 2007-2017, 2012

22. Muller PA and Vousden KH: Mutant p53 in cancer: New functions and therapeutic opportunities. Cancer Cell 25: 304-317, 2014.

23. Yeudall WA, Vaughan CA, Miyazaki H, Ramamoorthy M, Choi MY, Chapman CG, Wang H, Black E, Bulysheva AA, Deb SP, et al: Gain-of-function mutant 553 upregulates CXC chemokines and enhances cell migration. Carcinogenesis 33 442-451, 2012
24. Haupt Y, Maya R, Kazaz A and Oren M: Mdm2 promotes the rapid degradation of p53. Nature 387: 296-299, 1997.

25. Bond GL, Hu W and Levine AJ: MDM2 is a central node in the p53 pathway: 12 years and counting. Curr Cancer Drug Targets 5: 3-8, 2005.

26. Onel $\mathrm{K}$ and Cordon-Cardo C: MDM2 and prognosis. Mol Cancer Res 2: 1-8, 2004.

27. de Rozieres S, Maya R, Oren M and Lozano G: The loss of mdm2 induces p53-mediated apoptosis. Oncogene 19: 1691-1697, 2000

28. Steinman HA, Burstein E, Lengner C, Gosselin J, Pihan G, Duckett CS and Jones SN: An alternative splice form of Mdm2 induces p53-independent cell growth and tumorigenesis. J Biol Chem 279: 4877-4886, 2004

29. Terzian T, Suh YA, Iwakuma T, Post SM, Neumann M, Lang GA, Van Pelt CS and Lozano G: The inherent instability of mutant p53 is alleviated by Mdm2 or p16INK4a loss. Genes Dev 22: 1337-1344, 2008.

30. Livak KJ and Schmittgen TD: Analysis of relative gene expression data using real-time quantitative PCR and the 2(-delta delta C(T)) method. Methods 25: 402-408, 2001.

31. Vijayakumaran R, Tan KH, Miranda PJ, Haupt S and Haupt Y: Regulation of Mutant p53 Protein Expression. Front Oncol 5: 284,2015

32. Vousden KH and Prives C: Blinded by the light: The growing complexity of p53. Cell 137: 413-431, 2009.

33. Kojima K, Konopleva M, Samudio IJ, Schober WD Bornmann WG and Andreeff M: Concomitant inhibition of MDM2 and $\mathrm{Bcl}-2$ protein function synergistically induce mitochondrial apoptosis in AML. Cell Cycle 5: 2778-2786, 2006.

34. Li M, Brooks CL, Wu-Baer F, Chen D, Baer R and Gu W: Mono-versus polyubiquitination: Differential control of p53 fate by Mdm2. Science 302: 1972-1975, 2003.

35. Shadfan M, Lopez-Pajares V and Yuan ZM: MDM2 and MDMX: Alone and together in regulation of $\mathrm{p} 53$. Transl Cancer Res 1: 88-89, 2012.

36. Leslie PL, Ke H and Zhang Y: The MDM2 RING domain and central acidic domain play distinct roles in MDM2 protein homodimerization and MDM2-MDMX protein heterodimerization. J Biol Chem 290: 12941-12950, 2015.

37. Brooks CL and Gu W: p53 ubiquitination: $\mathrm{Mdm} 2$ and beyond. Mol Cell 21: 307-315, 2006.

38. Hoffman Y, Pilpel Y and Oren M: microRNAs and Alu elements in the p53-Mdm2-Mdm4 regulatory network. J Mol Cell Biol 6: 192-197, 2014.

39. Kamio T, Gu BW, Olson TS, Zhang Y, Mason PJ and Bessler M: Mice with a Mutation in the Mdm2 gene that interferes with MDM2/Ribosomal protein binding develop a defect in Erythropoiesis. PLoS One 11: e0152263, 2016. 